\title{
Genetic-Based New Fuzzy Reasoning Models with Application to Fuzzy Control
}

\author{
Daihee Park, Abraham Kandel, Fellow, IEEE, and Gideon Langholz, Senior Member, IEEE
}

\begin{abstract}
The successful application of fuzzy reasoning models to fuzzy control systems depends on a number of parameters, such as fuzzy membership functions, that are usually decided upon subjectively. It is shown in this paper that the performance of fuzzy control systems may be improved if the fuzzy reasoning model is supplemented by a genetic-based learning mechanism. The genetic algorithm enables us to generate an optimal set of parameters for the fuzzy reasoning model based either on their initial subjective selection or on a random selection. It is shown that if knowledge of the domain is available, it is exploited by the genetic algorithm leading to an even better performance of the fuzzy controller.
\end{abstract}

\section{INTRODUCTION}

QINCE the introduction of the basic methods of fuzzy Nreasoning by Zadeh [16], and the success of their first application to fuzzy control [9], the Fuzzy Reasoning Method (FRM) and its application to fuzzy control have been widely studied. However, certain challenging problems still remain open, including: 1) the completeness of the fuzzy rule base; 2) the subjective definitions of fuzzy subsets; and 3) the choice of fuzzy implication operators.

Recently, Cao et al. [1] have proposed a New Fuzzy Reasoning Method (NFRM) which turned out to be superior to Zadeh's FRM. Li [8] has applied the NFRM to the cart-pole system and compared it with the FRM, whereas Yu et al. [15] have applied it successfully to the control of an activated sludge plant.

Nevertheless, in the NFRM, the fuzzy relation matrix, determined by the human operator according to his experience, plays an important role, but may be difficult to extract optimally from the operator, particularly as the system increases in complexity. Moreover, whereas the selection of acceptable fuzzy membership functions is generally a subjective decision, a change in the membership functions may alter the performance of the fuzzy logic controller (FLC) significantly. Procyk and Mamdani [14] introduced an iterative procedure for altering membership

Manuscript received November 15, 1991; revised June 6, 1992 and February $27,1993$.

D. Park is with the Department of Computer Science, Korea University, Chochiwon, Korea.

A. Kandel is with the Department of Computer Science and Engineering, University of South Florida, Tampa, FL 33620.

G. Langholz is with the Department of Electrical Engineering, Florida State University, Tallahassee, FL 32306.

IEEE Log Number 9212926. functions to improve the performance of an FLC, but it is heuristic and still subjective.

The foregoing discussion indicates that a systematic procedure for the design of self-tuning fuzzy controllers is needed. It is shown in this paper that the performance of fuzzy control systems can be improved by incorporating a genetic-based learning mechanism into the NFRM. The genetic algorithm facilitates the derivation of the optimal fuzzy relation matrix and fuzzy membership functions based either on their initial subjective selection or on a random selection. It is shown that if knowledge of the domain is available, it can be exploited by the genetic algorithm leading to an even better performance of the fuzzy controller.

The paper is organized as follows. In Section II, we briefly introduce a dc series motor which is used as an example, albeit a simple one, of a controlled process. We evaluate the performance of the various FLC's discussed in this paper with respect to this process. In Section III, we discuss the FRM and NFRM controllers. In Section IV, we propose a genetic-based new fuzzy reasoning model which circumvents some drawbacks of the NFRM. Finally, Section V concludes with a brief summary.

\section{DC SERIES Motor}

A dc (direct current) series motor is an example of a simple, controlled process that can serve as a vehicle for the evaluation of the performance of the various controllers discussed in this paper.

In a dc series motor, the field winding is connected in series with the armature. Since the magnetic flux in the motor is proportional to the field current, which varies, the relationship between the current and the speed at which the motor's shaft rotates is nonlinear in general.

The particular dc series motor that we use in this paper is that investigated by Kiszka et al. [6], [7]. Fig. 1 reproduces the steady-state characteristic of the motor obtained by Kiszka and co-workers from actual measurements.

\section{Fuzzy Models}

\section{A. Fuzzy Reasoning Method}

A block diagram depicting the application of the Fuzzy Reasoning Method (FRM) [16] to process control is shown in Fig. 2. 


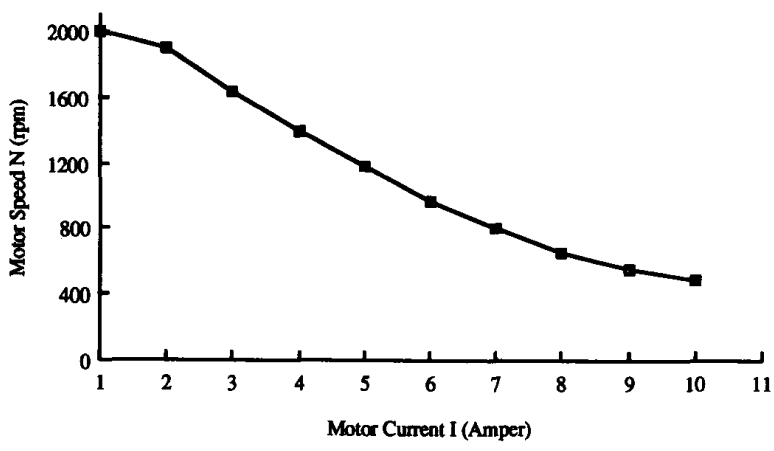

Fig. 1. Actual characteristic of the dc series motor. (Source: [8 Fig. 2.2] Reproduced by permission of Elsevier Science Publishers.)

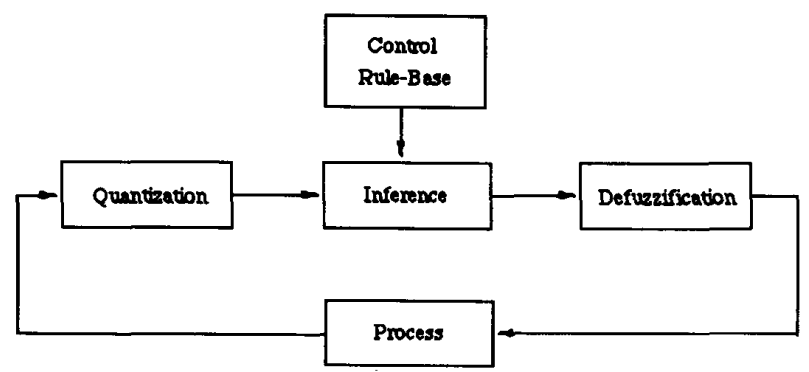

Fig. 2. Structure of a fuzzy rule-based control system [2].

Throughout this paper, we adopt the following fuzzy conditional statements to describe a certain situation:

IF $X$ is $A(1)$, THEN $Y$ is $B(1)$,

ALSO

IF $X$ is $A(2)$, THEN $Y$ is $B(2)$,

ALSO

\section{IF $X$ is $A(n)$ THEN $Y$ is $B(n)$}

where $X$ and $Y$ are two variables, and $A(1), \cdots, A(n)$ and $B(1), \cdots, B(n)$ are linguistic descriptions of $X$ and $Y$, respectively, which can be quantified by the fuzzy subsets $A_{i}^{*}$ and $B_{i}^{*}$ for each $i$ in the ranges of $X$ and $Y$. The fuzzy conditional statements (1) can be formalized in the form of the fuzzy relation $R(X, Y)$ [16]

$$
R(X, Y)=\text { ALSO }\left(R_{1}, R_{2}, \cdots, R_{i}, \cdots, R_{n}\right)
$$

where ALSO represents a sentence connective which combines the $R_{i}$ 's into the fuzzy relation $R(X, Y)$, and $R_{i}$ denotes the fuzzy relation between $X$ and $Y$ determined by the $i$ th fuzzy conditional statement, namely,

$$
R_{i}=\int \mu_{A^{*} i}(x) \otimes \mu_{B^{*} i}(y) /(x, y), \quad i=1,2, \cdots, n
$$

where $\otimes$ is a fuzzy implication operator.

Having established a fuzzy relation $R(X, Y)$ between two variables $X$ and $Y$, the compositional rule of inference

is then applied to infer the fuzzy subset $B$ for $Y$, given a fuzzy subset $A$ for $X$ :

$$
B=A \circ R(X, Y)
$$

where ${ }^{\circ}$ is a compositional operator.

In order to calculate the deterministic value of a linguistic variable $Y$, which is defined as a variable whose value is a sentence in a natural or artificial language, the following defuzzification method is applied [6]-[7]:

$$
y=\sum_{k=1}^{m} y_{k} / m
$$

where $y$ is a particular value of the variable $Y, y_{k}$ is the support value in which the membership function $\mu_{B^{*} k}(y)$ reaches its maximum grade of membership, and $m$ is the number of such support elements.

In Table I, we list several fuzzy implication operators which will be used in this paper. It should be noted that the sentence connective ALSO in the linguistic description is interpreted as intersection ( ${ }^{\wedge}$ i.e., minimum).

To see how the FRM can be applied in the context of a fuzzy rule-based control system (Fig. 2), let the controlled process be the dc series motor introduced in Section II. Observing the relationship between the armature current $I$ and the speed $N$ of the motor's shaft (Fig. 1), the process operator may formulate the following linguistic descriptions to specify the static characteristic of the motor (source: [6, eq. (2.2)]. Reproduced by permission of Elsevier Science Publishers):

IF $I=$ null

ALSO

IF $I=$ zero

ALSO

IF $I=$ small

ALSO

IF $I=$ medium

ALSO

IF $I=$ large

THEN $N=$ zero

\section{ALSO}

If $I=$ very large $\quad$ THEN $N=$ zero

where $I$ and $N$ are the linguistic variables corresponding to the current and speed, respectively. Notice that the first linguistic statement in (2) is not apparent in Fig. 1. It reflects the fact that, in a dc series motor, if the load is disconnected from the motor shaft, high speeds would result because of the small armature current that flows [2].

From the verbal descriptions (2), we see that the variable $I$ has six linguistic values: NULL, ZERO, SMALL, MEDIUM, LARGE, and VERY LARGE, whereas the variable $N$ has five: ZERO, SMALL, MEDIUM, LARGE, and VERY LARGE. The membership functions 
TABLE I

FUZZY IMPLICATION OPERATORS

$$
\begin{aligned}
& R_{2}=\int_{U X V}\left[\mu_{A}(a) \rightarrow_{2} \mu_{B}(b)\right] /(a, b) \\
& R_{4}=\int_{U x V}\left[1^{\wedge}\left(\mu_{B}(b) / \mu_{A}(a)\right)\right] /(a, b) \\
& R_{5}=\int_{U x V}\left[1^{\wedge}\left(1-\mu_{A}(a)+\mu_{B}(b)\right)\right] /(a, b) \\
& R_{27}=\int\left[\mu_{A}(a) \rightarrow_{27} \mu_{B}(b)\right] /(a, b) \\
& \text { where } \mu_{A}(a) \rightarrow_{2} \mu_{B}(b)=1 \text { if } \mu_{A}(a) \leq \mu_{B}(b) \\
& =0 \text { otherwise } \\
& R_{28}=\int_{U \times V}\left[1^{\wedge}\left[\mu_{B}(b) *\left(1-\mu_{A}(a)\right) /\left(\mu_{A}(a) *\left(1-\mu_{B}(b)\right)\right]\right] /(a, b)\right. \\
& \text { where } \mu_{A}(a) \rightarrow_{27} \mu_{B}(b)=1 \quad \text { if } \mu_{A}(a) \leq \mu_{B}(b) \\
& =\mu_{B}(b) \text { otherwise }
\end{aligned}
$$

Notes: $a$ and $b$ are particular values of the variables $A$ and $B$, respectively. $\mu_{A}(a)$ and $\mu_{B}(b)$ are the grades of membership of $a$ and $b$, respectively. $\mathbf{x}$ denotes the Cartesian product and $*$ denotes the arithmetic product. $U$ and $V$ are the universes of discourse of $A$ and $B$, respectively.

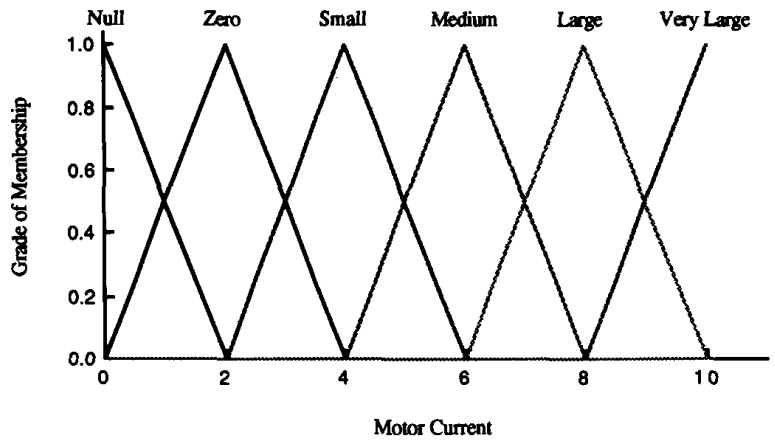

Fig. 3. Membership functions of the fuzzy subsets for motor current $I$.

of the fuzzy subsets for the motor current $I$ and speed $N$ are shown in Figs. 3 and 4, respectively.

Fig. 5 shows the results obtained by Kiszka et al. [6][7] using the implication operator $R_{2}, R_{4}, R_{5}, R_{27}$, or $R_{28}$ (see Table I). These operators resulted in the smallest mean square error, $e^{2}$, used to estimate the discrepancy between the motor's actual (measured) characteristic (Fig. 1) and that produced through the application of the FRM, where

$$
e^{2}=\sum_{i=1}^{v}\left(n_{r i}-n_{m i}\right)^{2} / \sum_{i=1}^{v} n_{r i}^{2}
$$

Here, $n_{r i}$ is the actual speed value for some current value $i, n_{m i}$ is the corresponding FRM-produced value, and $v$ is the number of discretization intervals of $N$.

While the FRM offers model-free estimation of the control system characteristics, and may prove more robust and easier to modify than a mathematical model of the process, the results obtained depend on the number of factors, such as: 1) the completeness of the fuzzy rule base; 2) the subjective definitions of the fuzzy subsets; 3 ) the choice of fuzzy implication operators; and 4) the defuzzification procedure that calculates the deterministic value of a fuzzy set.

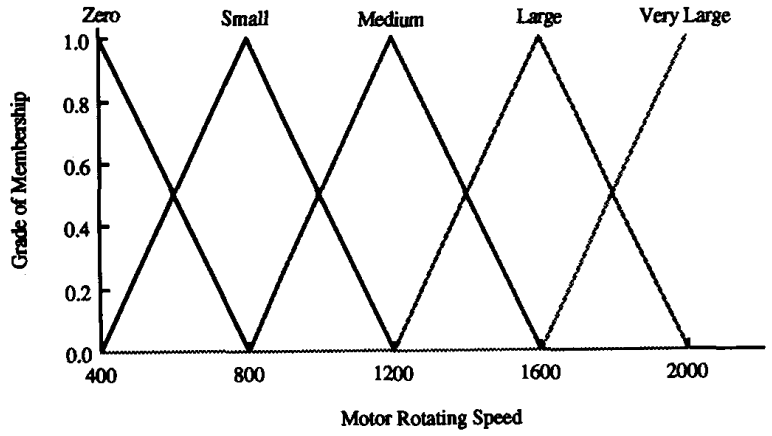

Fig. 4. Membership functions of the fuzzy subsets for motor speed $N$.

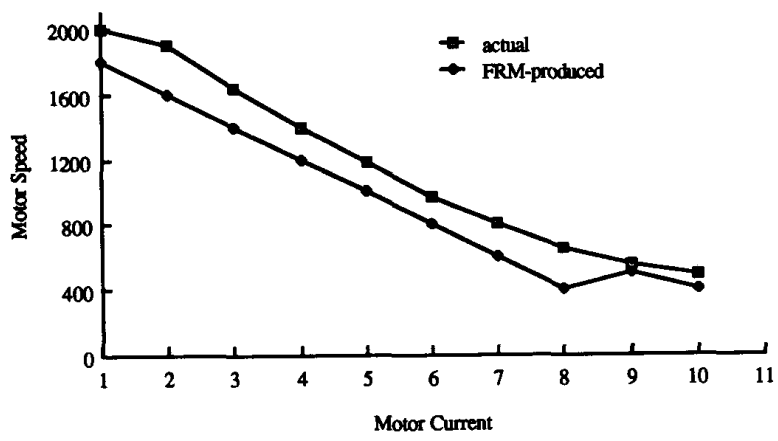

Fig. 5. Actual and FRM-produced motor characteristic $\left(e^{2}=2.504 *\right.$ $\left.10^{-2}\right)$.

\section{B. New Fuzzy Reasoning Method}

The New Fuzzy Reasoning Method (NFRM) [1] is shown schematically in Fig. 6 . In this figure, $x=\left[x_{1}, x_{2}\right.$, $\left.\cdots, x_{n}\right]$ is a vector consisting of the membership degrees of the real-valued input $x, y=\left[y_{1}, y_{2}, \cdots, y_{m}\right]$ is a vector consisting of the membership degrees of the output variable $y$, and $\boldsymbol{R}$ is an $n \times m$ fuzzy relation matrix whose elements $w_{i j}(i=1,2, \cdots, n ; j=1,2, \cdots$, $m$ ) indicate the relation degree between the $i$ th linguistic description for $X$ and the $j$ th linguistic description for $Y$. 


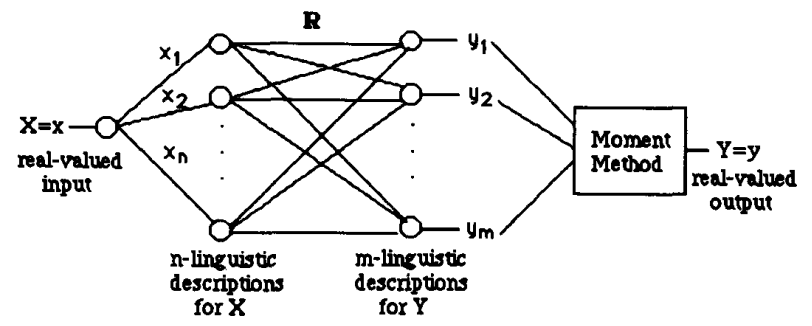

Fig. 6. NRFM schema

The $y_{i}$ 's are determined by

$$
y_{i}=\sum_{j} x_{j} * w_{j i}
$$

whereas the real number $y$ in the universe of $Y$ is evaluated using the moment method [1]:

$$
y=\sum_{j}\left(f_{j} * y_{j}\right) / \sum_{j} y_{j}
$$

where the $f_{j}$ 's are the central values of the membership functions of the linguistic descriptions for $Y$.

The NFRM algorithm can be described as follows.

1) Determine the linguistic descriptions of the input variable $X$ and the output variable $Y$, and their membership functions.

2) For a given real-valued input $X=x$, determine the membership degree corresponding to each linguistic description of $X$ by means of the fuzzy membership functions. That is, obtain $x=\left[x_{1}, \cdots, x_{n}\right]$ where $x_{i}$ is the membership degree of the $i$ th linguistic description of $X$.

3) Determine the fuzzy relation matrix $R$ between the linguistic descriptions of $X$ and $Y$. These relations are based on the expert's knowledge which is given in terms of linguistic descriptions.

4) Calculate the vector $y=\left[y_{1}, y_{2}, \cdots, y_{m}\right]$ in terms of the vector $\boldsymbol{x}$ and the fuzzy relation matrix $R$ :

$$
\boldsymbol{y}=\boldsymbol{x} \circ \boldsymbol{R} .
$$

It should be noted that, throughout this paper, we use the sum-of-product operator for the compositional operator in the NFRM.

5) Using the moment method (4), transform the vector $y$ to the corresponding real number $y$ in the universe of the variable $Y$.

To illustrate the application of the NFRM to the dc series motor, we consider two fuzzy relation matrices $\boldsymbol{R}_{1}$ and $\boldsymbol{R}_{\mathbf{2}}$ (shown in Tables II and III, respectively) commensurate with the linguistic statements (2). The results are shown in Figs. 7 and 8, respectively. Judging by the mean-square error, we see that the choice of Table II is a bad one relative to that of Table III.

As indicated by the mean square error in these examples, it seems that the NFRM controller outperforms the FRM controller. In addition, although not shown here, the NFRM controller requires less time than the FRM controller to execute the control tasks. Nevertheless, the results obtained through the application of the NFRM still
TABLE II

FUZZY RELATION MATRIX $\boldsymbol{R}_{1}$

\begin{tabular}{lccccc}
\hline \multicolumn{1}{c}{ I\N } & Zero & Small & Medium & Large & Very Large \\
\hline Null & 0.0 & 0.0 & 0.0 & 0.0 & 1.0 \\
Zero & 0.0 & 0.0 & 0.0 & 1.0 & 0.0 \\
Small & 0.0 & 0.0 & 1.0 & 0.0 & 0.0 \\
Medium & 0.0 & 1.0 & 0.0 & 0.0 & 0.0 \\
Large & 1.0 & 0.0 & 0.0 & 0.0 & 0.0 \\
Very Large & 1.0 & 0.0 & 0.0 & 0.0 & 0.0 \\
\hline
\end{tabular}

TABLE III

FUZZY RELATION MATRIX $\boldsymbol{R}_{2}$

\begin{tabular}{lccccc}
\hline \multicolumn{1}{c}{$I \backslash N$} & Zero & Small & Medium & Large & Very Large \\
\hline Null & 0.0 & 0.0 & 0.0 & 0.0 & 1.0 \\
Zero & 0.0 & 0.0 & 0.0 & 0.5 & 0.9 \\
Small & 0.0 & 0.0 & 0.5 & 0.9 & 0.3 \\
Medium & 0.5 & 1.0 & 0.2 & 0.0 & 0.0 \\
Large & 1.0 & 0.5 & 0.0 & 0.0 & 0.0 \\
Very Large & 1.0 & 0.2 & 0.0 & 0.0 & 0.0 \\
\hline
\end{tabular}

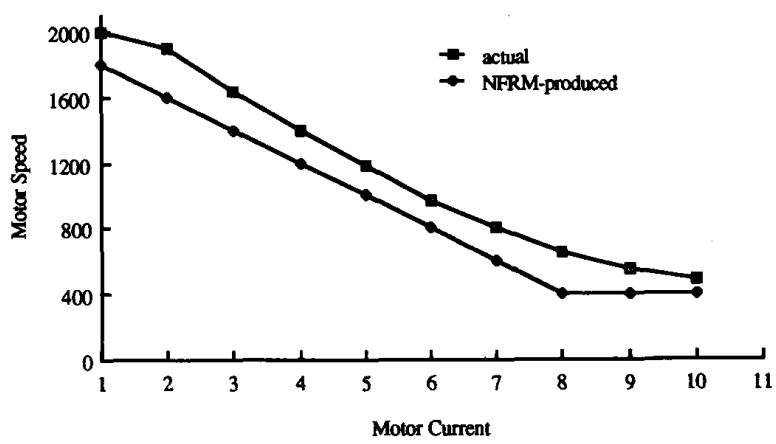

Fig. 7. Actual and NFRM-produced motor characteristic corresponding to $\boldsymbol{R}_{1}$ of Table $2\left(e^{2}=2.633 * 10^{-2}\right)$



Fig. 8. Actual and NRFM-produced motor characteristic corresponding to $\boldsymbol{R}_{\mathbf{2}}$ of Table III $\left(e^{2}=8.556 * 10^{-3}\right)$.

depend on the same factors as in the FRM case, except for the choice of fuzzy implication operators, and in addition, they also depend on the choice of the fuzzy relation matrix $\boldsymbol{R}$. 


\section{Genetic-Based New Fuzzy Reasoning Models}

As was noted previously, the performance of NFRMbased controllers mainly depends on the subjective selection of the fuzzy relation matrix, and on the subjective definition of the fuzzy subsets.

One way in which these dependencies can be circumvented is by incorporating genetic learning mechanisms into the NFRM. In this section, first we introduce some rudimentary aspects of genetic algorithms, and then show how a genetic algorithm can be applied in the context of the NFRM. In particular, we show that the performance of fuzzy control systems can be improved by using a genetic algorithm to facilitate the derivation of the optimal fuzzy relation matrix and fuzzy membership functions.

\section{A. Genetic Algorithms}

Genetic algorithms (GA's) are powerful search and optimization algorithms, based on semblance of natural genetics, which can be characterized by the following features [11].

1) A scheme for encoding solutions (referred to as chromosomes) to the problem.

2) An evaluation function that rates each chromosome relative to the others in the current set of chromosomes (referred to as population).

3) An initialization procedure for the population of chromosomes.

4) A set of operators which are used to manipulate the genetic composition of the population.

5) A set of parameters that provide the initial settings for the algorithm and operators, as well as the algorithm's termination condition.

The terminology of genetic algorithms originated from natural genetics. A candidate solution is called a chromosome and consists of a linear list of genes, where each gene can assume a finite number of values (alleles). A population consists of a finite number of chromosomes. The genetic algorithm (GA) evaluates a population and generates a new one iteratively, with each successive population referred to as a generation. Given an initial population $G(0)$, the $\mathrm{GA}$ generates a new generation $G(t+1)$ based on the previous generation $G(t)$ as follows:

1) $t=0$

2) Generate an initial population $G(t)$;

3) Evaluate $G(t)$;

4) If some termination conditions are met, go to Step

5) Generate new generation $G(t+1)$ from $G(t)$;

6) Evaluate $G(t+1)$

7) Return to Step 4;

9) Stop.

The evaluation function that we use, by which the GA rates each chromosome relative to the others in the current generation, is given by

$$
J=1 /\left(1+e^{2}\right)
$$

where $e^{2}$ is the mean-square error defined in (3). This evaluation function assigns the maximal ranking to that chromosome whose selection yields the minimal mean square error for the NFRM controller. It should be noted that through this choice of evaluation function, namely, its dependency on $e^{2}$, the GA is incorporated into the NFRM so that the FLC performance can be maximized.

The GA uses three basic operators to manipulate the genetic composition of a population: reproduction, crossover, and mutation. Reproduction is a process by which the most highly rated chromosomes (in accordance with the evaluation function) in the current generation are reproduced (copied) in the new generation. The copies are entered into a mating pool for further genetic operations.

Crossover provides a mechanism for chromosomes to mix and match attributes through random processes. First, pairs of reproduced chromosomes (referred to as parents) from the mating pool are selected at random. Second, an arbitrary gene position (called the crossover site) is selected at random. Third, the genes following the crossover site are swapped between the two chromosomes in each pair, resulting in two new offsprings. For example, if the parents are represented by the vectors $\left[a_{1}, b_{1}, c_{1}\right.$, $\left.d_{1}, e_{1}\right]$ and $\left[a_{2}, b_{2}, c_{2}, d_{2}, e_{2}\right]$ and $\left[a_{2}, b_{2}, c_{2}, d_{1}, e_{1}\right]$.

The third step of the crossover operation may be applied to all pairs of parents, or it may be applied, as we do here, only to some selected pairs. In the latter case, the selection is determined by the crossover probability. For example, if the crossover probability is 0.9 , then $90 \%$ of the pairs are crossed, whereas the remaining $10 \%$ are added to the next generation without crossover.

Mutation is the occasional alteration of some gene values in a chromosome. Every gene in each chromosome is a candidate for mutation, and its selection is determined by the mutation probability. The mutation probability is kept usually at a low value to avoid losing a large number of good chromosomes. The current value of the selected gene is then replaced either by a random number or by a value that is either $110 \%$ or $90 \%$ of its current value by a $50 \%$ chance within the given range of values. In subsequent sections, the former is used in conjunction with the derivation of the fuzzy membership functions whereas the latter is used to derive the fuzzy relation matrix.

The parameter settings for the GA are summarized in Table IV. In most cases we base the initialization procedure of the GA on the initial selection of the fuzzy relation matrix and/or the fuzzy membership functions. In practice, these are usually subjective selections based on the human expert's interpretation of the linguistic variables. However, to show that such initial knowledge of the domain leads to an even better performance of the fuzzy controller, we compare in Section IV-B the results obtained by the GA when the initialization is based on prior knowledge with those obtained from random initialization.

The termination condition for the GA occurs when the maximum generation number (100 in our case; see Table IV) is reached. 
TABLE IV

Parameter Settings for the Ga

\begin{tabular}{lc}
\hline Population Size & 50 \\
Generation Number & 100 \\
Crossover Probability & 0.9 \\
Mutation Probability & 0.1 \\
\hline
\end{tabular}

The remainder of this section summarizes our results and is structured as follows: In Section IV-B, we use the GA to generate the optimal fuzzy relation matrix based on prior knowledge of it (for our purpose, we use $\boldsymbol{R}_{\mathbf{2}}$ of Table III for the initial selection), and also based on a random initial selection. The fuzzy membership functions used in Section IV-B are those shown in Figs. 3 and 4. In Section IV-C, we use the GA to generate the optimal fuzzy membership functions for the dc motor current $I$, based on an initial selection provided by the human expert (Fig. 3 represents the prior knowledge in this case). The fuzzy membership functions for the dc motor speed $N$ and the fuzzy relation matrix used in Section IV-C are those shown in Fig. 4 and Table III, respectively. Finally, in Section IV$\mathrm{D}$, we generate the optimal fuzzy relation matrix and fuzzy membership functions for the dc motor current by initializing the GA with prior knowledge provided in Table III and Fig. 3, respectively. The fuzzy membership functions for the dc motor speed used in Section IV-D are those shown in Fig. 4.

\section{B. Optimal Fuzzy Relation Matrix}

Since the fuzzy relation matrix used in conjunction with the dc motor is a $6 \times 5$ matrix, each chromosome is represented by an array of length 30 . Each gene corresponds to a fuzzy relation degree whose value is any real number between 0 and 1 .

The GA generates the optimal fuzzy relation matrix [in accordance with (5)] based on $\boldsymbol{R}_{2}$, the assumed prior knowledge provided by the human expert. The fuzzy membership functions used by the NFRM controller are those shown in Figs. 3 and 4.

The population of 50 chromosomes (see Table IV) is initialized by associating one of the chromosomes with the fuzzy relation matrix $\boldsymbol{R}_{\mathbf{2}}$ (Table III), whereas the remaining chromosome are randomly initialized from the range $[0,1]$. This guarantees that the performance of the GA-assisted NFRM controller will be at least as good as that of the original NFRM controller (Section III-B). As was mentioned before, mutation in this case is based on changing a selected gene's value to either $110 \%$ or $90 \%$ of its current value by a $50 \%$ chance within the given range $[0,1]$.

The resulting GA-generated fuzzy relation matrix and dc motor characteristic are shown in Table $\mathrm{V}$ and Fig. 9, respectively. As indicated by the mean square error, the performance of the NFRM controller with the GA-generated fuzzy relation matrix has improved significantly relative to the performance of the NFRM controller with
TABLE V

Ga-Generated Fuzzy Relation Matrix Based on $\boldsymbol{R}_{2}$ Initialization

\begin{tabular}{lccccc}
\hline \multicolumn{1}{c}{$I \backslash N$} & Zero & Small & Medium & Large & Very Large \\
\hline Null & 0.000 & 0.000 & 0.000 & 0.000 & 1.000 \\
Zero & 0.000 & 0.000 & 0.000 & 0.234 & 0.980 \\
Small & 0.000 & 0.000 & 0.868 & 0.422 & 0.139 \\
Medium & 0.190 & 0.430 & 0.752 & 0.000 & 0.000 \\
Large & 0.562 & 0.886 & 0.000 & 0.000 & 0.000 \\
Very Large & 1.000 & 0.287 & 0.000 & 0.000 & 0.000 \\
\hline
\end{tabular}

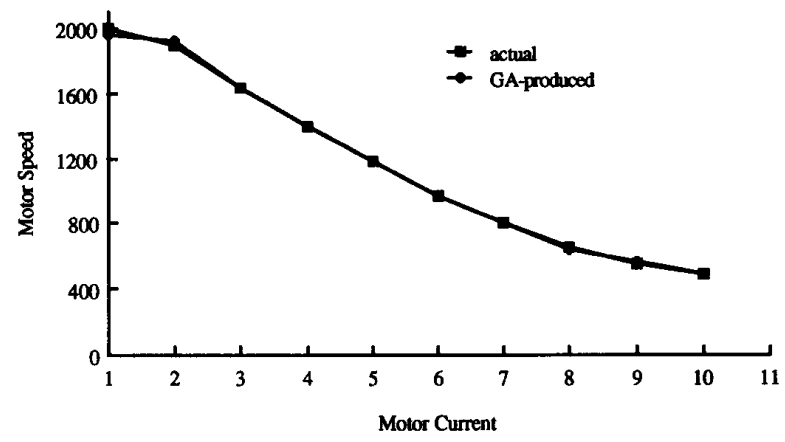

Fig. 9. Actual and GA-produced motor characteristic based on $\boldsymbol{R}_{\mathbf{2}}\left(e^{2}=\right.$ $\left.1.727 * 10^{-4}\right)$.

$\boldsymbol{R}_{2}$ as the fuzzy relation matrix (compare Fig. 9 with Fig. 8).

Rather than initialize the chromosomes as was mentioned above, consider now initializing the entire population randomly from the range $[0,1]$. The resulting GAgenerated fuzzy relation matrix and dc motor characteristic are shown in Table VI and Fig. 10, respectively. As can be seen, the mean square error in this case is an orderof-magnitude greater than that in Fig. 9 and is only slightly better than the mean-square error shown in Fig. 8 .

The difference in the results due to the two initialization procedures (one which is based on a priori knowledge, albeit subjective, and the other which is randomly based) seems to support the notion that prior knowledge enhances the performance of a GA-assisted NFRM controller. In addition, the convergence rate of the GA initialized with prior knowledge is markedly faster than that of the randomly initialized GA, as shown in Fig. 11.

\section{Optimal Fuzzy Membership Functions}

The design of FLC's has been, for the most part, a trialand-error process. In particular, Park et al., [12]-[13] have shown how the often subjective selection of fuzzy membership functions affects the performance of the FLC. However, they did not provide a systematic way to adjust the fuzzy membership functions. On the other hand, Procyk and Mamdani [14] introduced an iterative procedure for altering membership functions to improve the performance of an FLC, but it is heuristic and subjective.

The usefulness of GA's for learning fuzzy membership functions, so that FLC design can become more efficient, 
TABLE VI

Ga-Generated Fuzzy Relation Matrix Based on Random INITIALIZATION

\begin{tabular}{lccccc}
\hline \multicolumn{1}{c}{ I $\backslash \mathrm{N}$} & Zero & Small & Medium & Large & Very Large \\
\hline Null & 0.021 & 0.028 & 0.070 & 0.213 & 1.000 \\
Zero & 0.014 & 0.015 & 0.027 & 0.089 & 1.000 \\
Small & 0.104 & 0.130 & 0.066 & 1.000 & 0.025 \\
Medium & 0.681 & 0.485 & 0.013 & 0.132 & 0.366 \\
Large & 1.000 & 0.113 & 0.098 & 0.126 & 0.027 \\
Very Large & 1.000 & 0.161 & 0.050 & 0.034 & 0.034 \\
\hline
\end{tabular}

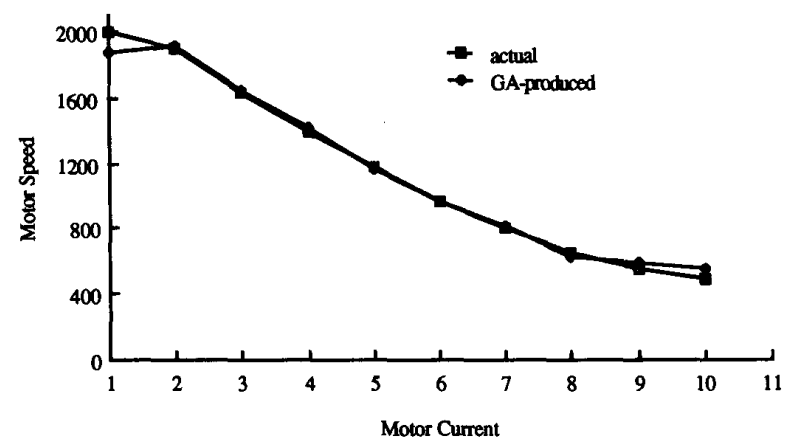

Fig. 10. Actual and GA-produced motor characteristic based on random initialization $\left(e^{2}=1.370 * 10^{-3}\right)$.

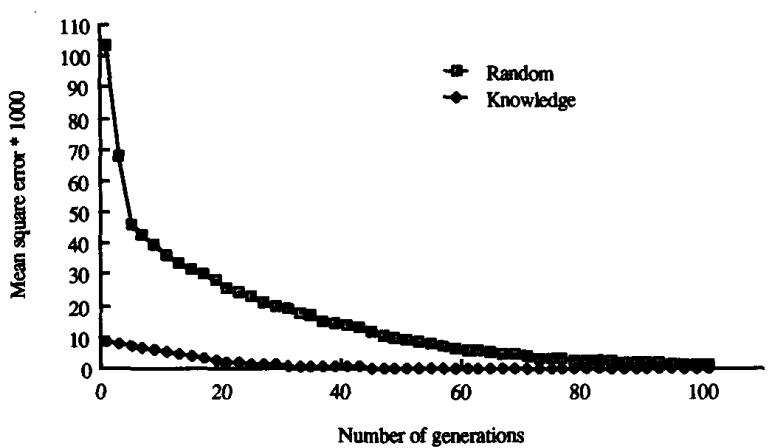

Fig. 11. Convergence rates of GA-knowledge and GA-random.

was already mentioned in the literature [5], [10]. In this section, we show the mechanics of generating optimal fuzzy membership functions in the context of the NFRM controller.

The membership functions for each fuzzy subset can be derived from a base function whose parameters may be adjusted to fit any specific membership function. This base function is defined as

$$
\begin{array}{ll}
f\left(x ; \alpha_{i}, \beta_{i}, \gamma_{i}\right)=0, & \text { if } x \leq \alpha_{i} \\
f\left(x ; \alpha_{i}, \beta_{i}, \gamma_{i}\right)=\left(x-\alpha_{i}\right) /\left(\beta_{i}-\alpha_{i}\right), & \text { if } \alpha_{i}<x \leq \beta_{i} \\
f\left(x ; \alpha_{i}, \beta_{i}, \gamma_{i}\right)=\left(x-\gamma_{i}\right) /\left(\beta_{i}-\gamma_{i}\right), & \text { if } \beta_{i}<x \leq \gamma_{i} \\
f\left(x ; \alpha_{i}, \beta_{i}, \gamma_{i}\right)=0, & \text { if } x>\gamma_{i}
\end{array}
$$

where $\alpha_{i}, \beta_{i}$, and $\gamma_{i}$ are parameters. For example, the membership function for the fuzzy subset "Small" in Fig.
3 is obtained from (6) by setting $\alpha_{\text {small }}=2, \beta_{\text {small }}=4$, and $\gamma_{\text {small }}=6$, yielding

$$
\begin{array}{ll}
f(x ; 2,4,6)=0, & \text { if } x \leq 2, \\
f(x ; 2,4,6)=0.5 *(x-2), & \text { if } 2<x \leq 4 \\
f(x ; 2,4,6)=-0.5 *(x-6) & \text { if } 4<x \leq 6 \\
f(x ; 2,4,6)=0, & \text { if } x>6
\end{array}
$$

Now, for each fuzzy subset $i$, we set up the following equations:

$$
\begin{aligned}
& \alpha_{i}:=\left(\alpha_{i}+\delta_{i}\right)-\eta_{i} \\
& \beta_{i}:=\left(\beta_{i}+\delta_{i}\right) \\
& \gamma_{i}:=\left(\gamma_{i}+\delta_{i}\right)+\eta_{i}
\end{aligned}
$$

where $\delta_{i}$ and $\eta_{i}$ are adjustment coefficients. The coefficient $\delta_{i}$ makes the $i$ th membership function shift to the right or to the left without changing its shape, whereas the coefficient $\eta_{i}$ makes the $i$ th membership function for $i$ th fuzzy subset shrink or expand.

In this section, we use the GA to find the optimal values of $\delta_{i}$ and $\eta_{i}$ for the fuzzy membership functions of the dc motor current $I$ according to the evaluation function (5). For the first and last fuzzy membership functions, we set $\mu_{\text {null }}(x)=1$ if $x \leq \beta_{\text {null }}$ and $\mu_{\text {verylarge }}(x)=1$ if $x \geq$ $\beta_{\text {very large }}$. The coefficients $\delta_{i}$ and $\eta_{i}$ are any real numbers taken from the range $[-1.5,1.5]$. Since there are two coefficients and six fuzzy membership functions for the motor current, the length of each chromosome is 12 .

The GA generates the optimal fuzzy membership functions [in accordance with (5)] for the dc motor current based on the membership functions in Fig. 3, the assumed prior knowledge provided by the human expert. The fuzzy membership functions for the motor speed and the fuzzy relation matrix used by the NFRM controller are those given in Fig. 4 and Table III, respectively.

The population of 50 chromosomes (see Table IV) is initialized by setting all the genes of one of the chromosomes to 0 (corresponding to the fuzzy membership functions defined in Fig. 3), whereas the remaining chromosomes are initialized randomly from the range $[-1.5$, 1.5]. This guarantees that the performance of the GA-assisted NFRM controller will be at least as good as that of the original NFRM controller (Section III-B). As was mentioned in Section IV-A, mutation in this case is based on changing the selected gene's value randomly within the range $[-1.5,1.5]$.

The resulting GA-generated fuzzy membership functions for the motor current and the dc motor characteristic are shown in Figs. 12 and 13, respectively. We see that the mean square error in this case is slightly worse than that of Fig. 9, but it is an order-of-magnitude smaller than the mean square error shown in Fig. 8.

\section{Simultaneous Derivation of Optimal Fuzzy Relation Matrix and Fuzzy Membership Functions}

In this section, we use the GA to find simultaneously the optimal fuzzy relation matrix and the fuzzy member- 


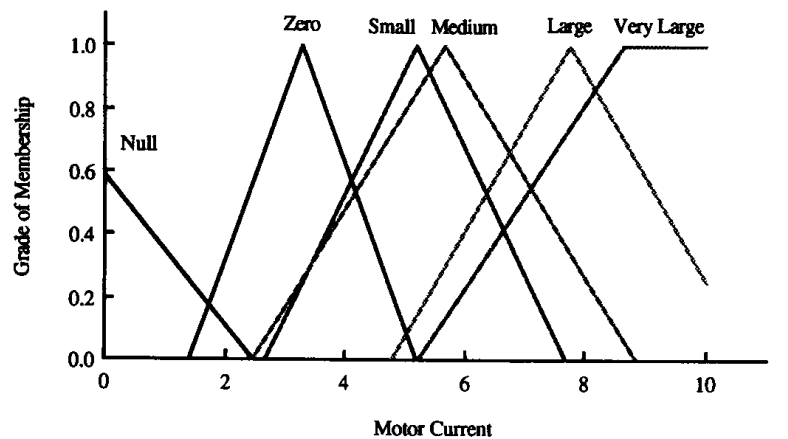

Fig. 12. GA-generated fuzzy membership functions for motor current $I$.

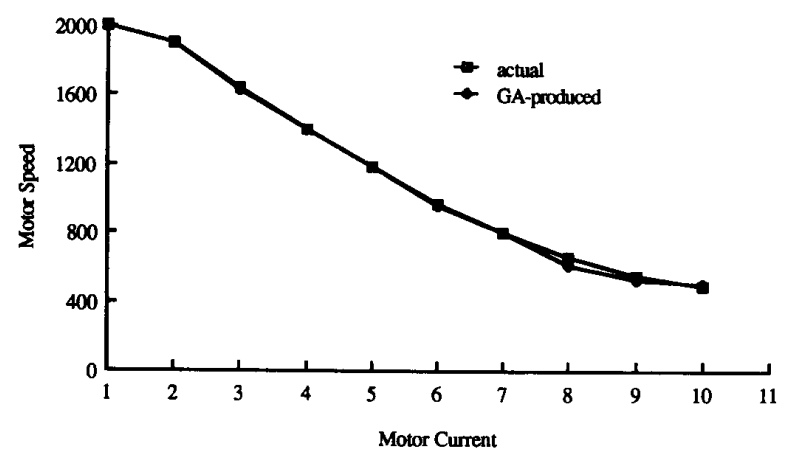

Fig. 13. Actual and GA-produced motor characteristic $\left(e^{2}=2.009 *\right.$ $\left.10^{-4}\right)$.

ship functions for the dc motor current $I$. These are generated based on $\boldsymbol{R}_{\mathbf{2}}$ (Table III) and Fig. 3, which represent the assumed prior knowledge provided by the human expert. The fuzzy membership functions for the motor speed used by the NFRM controller are those given in Fig. 4 .

Each chromosome consists of an array of length 42 . The first 30 genes are allocated to the fuzzy relation matrix, the genes values being any real number between 0 and 1 (see Section IV-B). The next 12 genes are allocated to the fuzzy membership functions of the motor current, the genes' values being any number from the range $[-1.5$, 1.5] (see Section IV-C). Since each chromosome consists of two different subchromosomes (one for the fuzzy relation matrix and the other for the fuzzy membership functions), we treat the two subchromosomes as independent entities as far as crossover and mutation are concerned, but treat both as a single entity as far as the reproduction operator is concerned.

The population of 50 chromosomes (see Table IV) is initialized so that one of the chromosomes inherits the prior knowledge given in Table III and Fig. 3, whereas the remaining chromosomes are initialized at random.

The resulting GA-generated optimal fuzzy relation matrix and fuzzy membership functions are shown in Table VII and Fig. 14, respectively, and the de motor characteristic is shown in Fig. 15. Judging by the mean square error, the result in this case is the best thus far.
TABLE VII

NEW uZZy Relation Matrix

\begin{tabular}{lccccc}
\hline \multicolumn{1}{c}{$\mathrm{I} \backslash \mathrm{N}$} & Zero & Small & Medium & Large & Very Large \\
\hline Null & 0.000 & 0.000 & 0.000 & 0.000 & 0.891 \\
Zero & 0.000 & 0.000 & 0.000 & 0.113 & 0.900 \\
Small & 0.000 & 0.000 & 0.868 & 0.650 & 0.241 \\
Medium & 0.495 & 0.891 & 0.192 & 0.000 & 0.000 \\
Large & 0.722 & 0.593 & 0.000 & 0.000 & 0.000 \\
Very Large & 0.882 & 0.266 & 0.000 & 0.000 & 0.000 \\
\hline
\end{tabular}

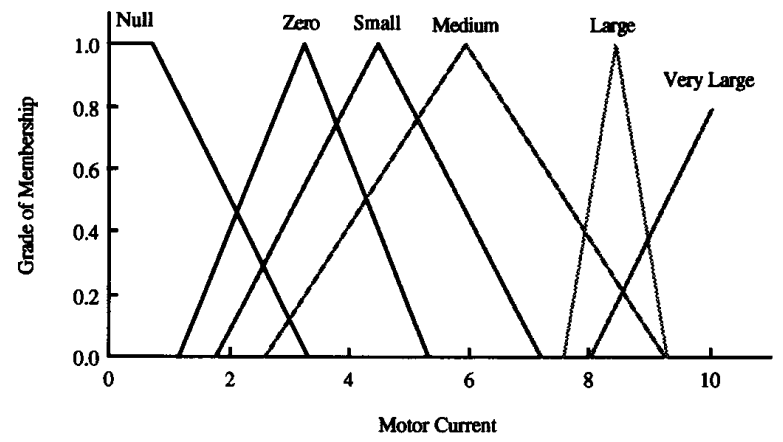

Fig. 14. New fuzzy membership functions for motor current $I$.



Fig. 15. Actual and GA-produced characteristic $\left(e^{2}=5.68 * 10^{-6}\right)$.

\section{ConClusion}

In this paper, we studied several fuzzy controllers and analyzed their performance using a dc series motor as the controlled process. We have shown that the performance of NFRM controllers can be enhanced significantly by including a genetic algorithm in the loop whose function is to search for optimal fuzzy relation matrix and/or fuzzy membership functions. It was shown that better results are obtained if the search procedure is initialized from prior knowledge provided by an expert. Consequently, by incorporating genetic algorithm learning mechanism into the NFRM we can alleviate some of the drawbacks associated with the NFRM, in particular, its dependence on the subjective fuzzy relation matrix and the subjective definition of the fuzzy subsets.

Our results indicate that the best performance of the NFRM controller is obtained when the space of both the 
fuzzy membership functions and the fuzzy relation matrix is searched by the genetic algorithm. As far as the fuzzy membership functions are concerned, other researchers have already established that their choice can affect significantly the performance of fuzzy controllers. Our results seem to lend additional support to this observation. In addition, in the context of NFRM controllers, we see that the fuzzy relation matrix also plays an important role in terms of the performance of the fuzzy controller.

Further investigation is required to study the space-time complexity of the controller algorithm. The particular pertinent issues are the time required for the algorithm to run, and scaling-up the algorithm to problems of greater complexity.

\section{REFERENCES}

[1] Z. Cao, A. Kandel, and L. Li, "A new model of fuzzy reasoning," Fuzzy Sets and Syst., vol. 36, pp. 311-325, 1990.

[2] V. Del Toro, Electric Machines and Power Systems. Englewood Cliffs, NJ: Prentice-Hall, 1985.

[3] J. Efstathiou, Expert Systems in Process Control, Inst. Meas. and Contr., England, 1989.

[4] A. Kandel and G. Langholz, Fuzzy Control Systems. Boca Raton, FL.: CRC Press, 1994.

[5] C. L. Karr and D. A. Stanley, "Fuzzy logic and genetic algorithms in time-varying control problems," in Proc. NAFIPS-91, 1991, pp. $285-290$.

[6] J. B. Kiszka, M. E. Kochanska, and D. S. Sliwinska, "The influence of some fuzzy implication operators on the accuracy of a fuzzy model, Part I," Fuzzy Sets and Syst., vol. 15, pp. 111-128, 1985.

[7] - " "The influence of some fuzzy implication operators on the accuracy of a fuzzy model, Part II,' Fuzzy Sets and Syst., vol. 15, pp. 223-240, 1985.

[8] L. Li, Fuzzy Inference and Its Applicability to Control Systems, Master's thesis. Florida State Univ., Tallahassee, 1989.

[9] E. H. Mamdani, "Application for fuzzy algorithms for the control of a dynamic plant,"' Proc. IEEE., vol. 121, pp. 1585-1588, 1974.

[10] D. L. Meredith, K. K. Kumar, and C. L. Karr, "The use of genetic algorithms in the design of fuzzy logic controllers," in Proc. WNNAIND 91, 1991, pp. 695-702.

[11] D. J. Montana and L. Davis, "Training feedforward neural networks using genetic algorithms," in Proc. IJCAI-89, 1989, pp. 762-767.

[12] D. Park, Z. Cao, and A. Kandel, "Investigations on the applicability of fuzzy inference,"' Fuzzy Sets and Systems, vol. 49, no. 2, pp. 151170,1992

[13] D. Park, A. Kandel, and G. Langholz, "Hybrid Neural-Fuzzy reasoning model with application to fuzzy control," in Fuzzy Control Systems, A. Kandel and G. Langholz, Editors. Boca Raton, FL.: CRC Press, 1994.

[14] T. J. Procyk and E. H. Mamdani, "A linguistic self-organizing process controller," Automatica, vol. 15, pp. 15-30, 1979.
[15] C. Yu, Z. Cao, and A. Kandel, "Application of fuzzy reasoning to the control of an activated sludge plant," Fuzzy Sets and Syst., vol. 38 , pp. $1-14,1990$

[16] L. A. Zedeh, "Outline of a new approach to the analysis of complex systems and decision processes," IEEE Trans. Syst., Man, Cybern., vol. SMC3, pp. 28-44, 1973.

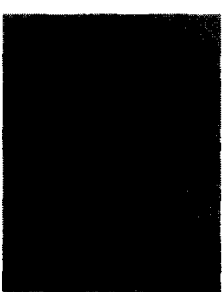

Daihee Park received the B.S. and M.S. degrees in mathematics from Korea University, Seoul, Korea, in 1982 and 1984 , respectively. He also received the M.S. and Ph.D. degrees in computer science from Florida State University in 1989 and 1992 , respectively.

Currently, he is an Assistant Professor in the Department of Computer Science, Korea University, Chochiwon. His research interests encompass neural networks, fuzzy logic, genetic algorithms, and machine learning.



Abraham Kandel (S'68-M'74-SM'79-F'92) received the B.Sc. degree in electrical engineering from the Technion, Israel Institute of Technol ogy, the M.Sc. degree from the University of New Mexico.

He is a Professor and Endowed Eminent Scholar in Computer Science and Engineering, and is the Chairman of the Department of Computer Science and Engineering at the University of South Flor ida. Previously, he was Professor and Chairman of the Computer Science Department at Florida State University, as well as the Director of the Institute of Expert Systems and Robotics at FSU and the Director of the State University System Center for Artificial Intelligence.

Dr. Kandel is a member of the ACM, and an Advisory Editor to the international journals Fuzzy Sets and Systems, Information Sciences, Expert Systems, and Engineering Applications of Artificial Intelligence. He has published over 250 research papers and 19 books.

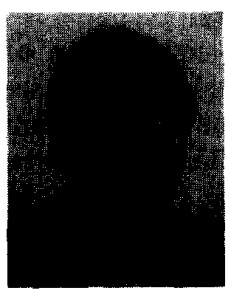

Gideon Langholz (S'68-M'71-SM'90) received the B.Sc. degree in electrical engineering from the Technion, Israel Institute of Technology, and the Ph.D. degree from the University of London, both in Electrical Engineering.

He is a Professor of Electrical Engineering at Florida State University and at Tel-Aviv University.

Dr. Langholz is a member of the Editorial Board of the International journal Engineering Applications of Artificial Intelligence. He has published over 60 research papers and three books. 